\title{
COORDINATED STASIS: INFORMING EXPECTATIONS AND NULL MUDDLES
}

SCHOPF*, Kenneth M., Museum of Comparative Zoology \& Dept. of Earth and Planetary Sciences, Harvard University, 26 Oxford Street, Cambridge, MA 02138, USA.; BAUMILLER, Tomasz K., Dept. of Earth and Planetary Sciences, Harvard University, 26 Oxford Street, Cambridge, MA 02138, USA.

The recent interest in whole-fauna dynamics generated by the documentation of "coordinated stasis" by Brett \& Baird has led to several realizations. First, that coordinated stasis may be equated with (indeed is expressed through) biofacies- very common features of the fossil record. Second, that in order to determine how these 3-7 MY blocks of faunal coherence fit into our world views of evolution and ecology (as predicted outcomes, or perhaps as counter-intuitive jolts) we require a sound understanding of our fields' neutral expectations for long-term faunal behavior.

The dynamics around which coordinated stasis revolves may be separated into stability (the norm) and change (the passing event), expressed in both the ecological and evolutionary histories of the fauna as in this table:

\begin{tabular}{|c|c|c|}
\hline$\underline{\text { Change }}$ & $\begin{array}{c}\text { Ecological } \\
\text { Reshuffling/ } \\
\text { Additions/subtractions }\end{array}$ & $\begin{array}{c}\text { Evolutionary } \\
\text { Turnover } \\
\text { (Speciation/Extinction) }\end{array}$ \\
\hline Stability & Coherent associations & Range Persistence \\
\hline
\end{tabular}

Thus far, the evolutionary aspects have been more amenable to null modeling approaches. Attempts to simulate the patterns of stratigraphic ranges consistent with those described as coordinated stasis by computer revealed that under two extinction scenarios, time-homogenous and exponential, coordinated stasis-like patterns could not be produced, while under an episodic extinction scenario, such patterns could be generated approximately $20 \%$ of the time. But coordinated stasis, and the crux of many of the points it has raised, lie in the ecological aspects of the pattern.

Null models of Recent faunal dynamics have been the source of much debate in the ecological literature. There appear to be few "rules" of behavior that are above objections of subjectivity. Whether the obstacles debated by ecologists disappear over geologic timescales, or are actually accentuated is an open question. The one certainty is that researchers seem less willing to ascribe a simple number to something that seems more concrete/observable than they are when dealing with the vagaries of evolution.

A conceptual model, based on four neutral premises (ecologicalorganismal subtractions and additions are possible, multiple stable states are available if triggered; evolutionary-allopatric speciation, and stabilizing selection) may serve as a touchstone for our intuitions about how our fields expect faunas to behave over long timescales. While coordinated stasis may be produced using this set of minimal rules, there are a number of caveats that must be made along the way. It is these caveats, the "gaps" between our neutral frameworks and biofacies/coordinated stasis that should inform us about the areas to which we should pay most of our attention: most notably how well environmental stability and faunal stability conform. 